\title{
A Phase Space Perspective on Electron Holography - Building Bridges Between Inline-, Off-axis Holography, Differential Phase Contrast and Diffractive Imaging.
}

\author{
Axel Lubk ${ }^{1}$, Falk Röder ${ }^{1}$ and Hannes Lichte ${ }^{1}$ \\ 1. Triebenberg Laboratory, Institute of Structure Physics, TU Dresden, 01062 Dresden, Germany.
}

Electron Holography (EH) was originally invented by D. Gabor in an attempt to correct the insurmountable spherical aberration of the objective lens in a Transmission Electron Microscope (TEM). The holographic principle proved so general that it found its way into a wide variety of techniques all aiming at reconstructing the phase information in a wave field of a particular radiation (not necessarily electrons) in one or the other way. As realized by D. Gabor, holography, in its classical appearance, is essentially nonlinear, that is, the reconstruction of a complex wave function $\Psi$ from image intensities $I$ (almost) necessarily leads to some sort of root extraction. The associated ambiguities manifest as twin or shadow images, non-converging reconstruction algorithms or non-unique reconstructions, depending on the holographic technique. Further difficulties arise in the presence of partial coherence, eventually obscuring the reconstruction of a single wave function (or its phase).

In an attempt to surmount these issues, a number of linear reconstruction schemes avoiding the issues of nonlinearity and partial coherence have been developed [1]. These so-called quantum state measurements seek a reconstruction of an object more general than the wave function, i.e., some representation of the density operator or elements of which. Here, we elaborate on this change of perspective, that is, we consider $\mathrm{EH}$ as a means to reconstruct the electron beam's quantum mechanical phase space distribution instead of its phase [2]. We thereby obtain a unified description of various holographic methods including related concepts such as superresolution. The unified framework allows for discussing the requirements (e.g., in terms of coherence), area of application (e.g., in terms of object properties) and generalizations (e.g., towards inelastic holography) of different types of EH, such as inline EH, off-axis EH or differential phase contrast (DPC). We particularly exploit the intuitive concept of phase space to visualize the various considerations as phase space diagrams.

The central object of our considerations is the quantum mechanical density operator $\hat{\rho}$ of the beam electrons in its various equivalent representations, namely the density matrix $\rho\left(x, x^{\prime}\right)$, the Wigner function $W(r, k)$ and the ambiguity function $\widetilde{W}(q, p)$ - all related by Fourier transforms along some axis (Fig. 1). Whereas the density matrix of a fully coherent wave function simply reads $\rho\left(x, x^{\prime}\right)=$ $\Psi(x) \Psi^{*}\left(x^{\prime}\right)$, its generalization towards incoherent mixtures, $\rho\left(x, x^{\prime}\right)=\sum_{n} P_{n} \Psi_{n}(x) \Psi_{n}^{*}\left(x^{\prime}\right)=$ $\iint \mathrm{d} x \mathrm{~d} x^{\prime} \rho\left(x, x^{\prime}\right)|x\rangle\left\langle x^{\prime}\right|$, allows for taking into account the effects of partial coherence introduced by the gun or inelastic scattering on the object. Most notably, the image intensity is $-I(r)=\rho\left(x=r, x^{\prime}=\right.$ $r)=\int \mathrm{d} k W(r, k)=\mathcal{F}\{\widetilde{W}(q, p=0)\}$ - the main diagonal, momentum projection or Fourier transformed zero section in the corresponding representation. Moreover, we show that, both, coherent and incoherent aberrations, affect the different representations through particular linear operations [3], such as convolutions, which may be (partially) inverted (i.e., corrected) in case of a complete phase space reconstruction.

Fig. 2 exhibits the generalized linear phase space reconstruction schemes of off-axis $\mathrm{EH}$ and inline (focal series) EH. Accordingly, off-axis EH corresponds to some sort of shift series [4], inline EH to a 
tilt series [5] and (generalized) DPC (not shown) to a Taylor expansion [6] in reciprocal phase space (ambiguity function). All of which can be used to assemble the complete phase space distribution provided that enough measurements (e.g., over a sufficient defocus range) have been performed.

The above considerations form the basis for our experimental phase space reconstructions of (in)elastically scattered electrons in the framework of off-axis EH, inline EH and (generalized) DPC. As a consequence of persisting instrumental limitations, such limited astigmatic defocus ranges, the reconstructions are restricted, e.g., to objects homogeneous in one spatial direction (systems of thin films). Nevertheless, we are able to extract, for instance, a restricted phase space distribution of plasmon scattered electrons, thereby measuring object properties inaccessible by simple intensity measurements. In summary, we can therefore show that the phase space perspective provides both, a unified view on different holographic techniques and a novel route towards object characterization within the TEM.

[1] M. Paris and J. Rehacek. Quantum State Estimation, Springer (2004).

[2] M. Testorf and A.W. Lohmann. Appl. Opt. (2008), 47, A70-A77.

[3] A. Lubk and F. Röder, Ultramicroscopy (2015).

[4] F. Röder and A. Lubk, Ultramicroscopy (2014), 2014, 146, 103 - 116.

[5] M. G. Raymer, M. Beck, D. McAlister, Phys. Rev. Lett. (1994), 72, 1137-1140.

[6] A. Lubk and J. Zweck, Phys. Rev. A (2015), 91, 023805.

[7] We acknowledge funding from the 17th round of the DIP Programme of the DFG.

ambiguity function density matrix Wigner function

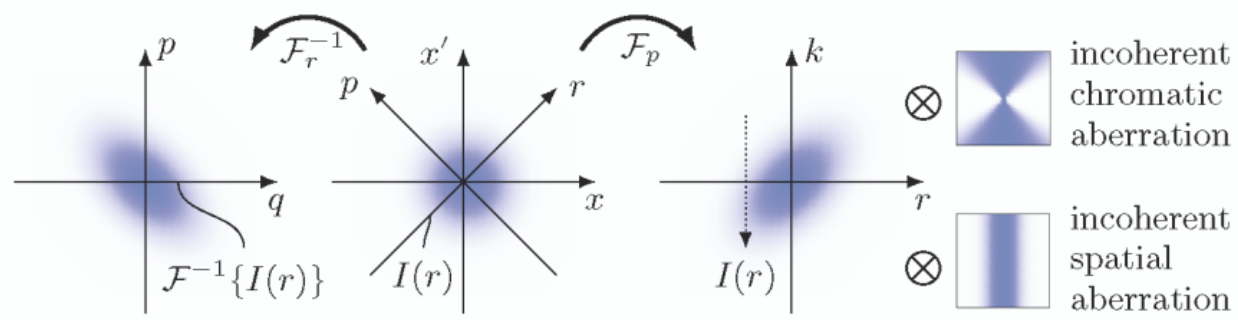

Figure 1. Different equivalent representations of the density operator of a Gaussian beam. The modifications (convolutions) of the Wigner function by spatial and temporal incoherence are indicated.

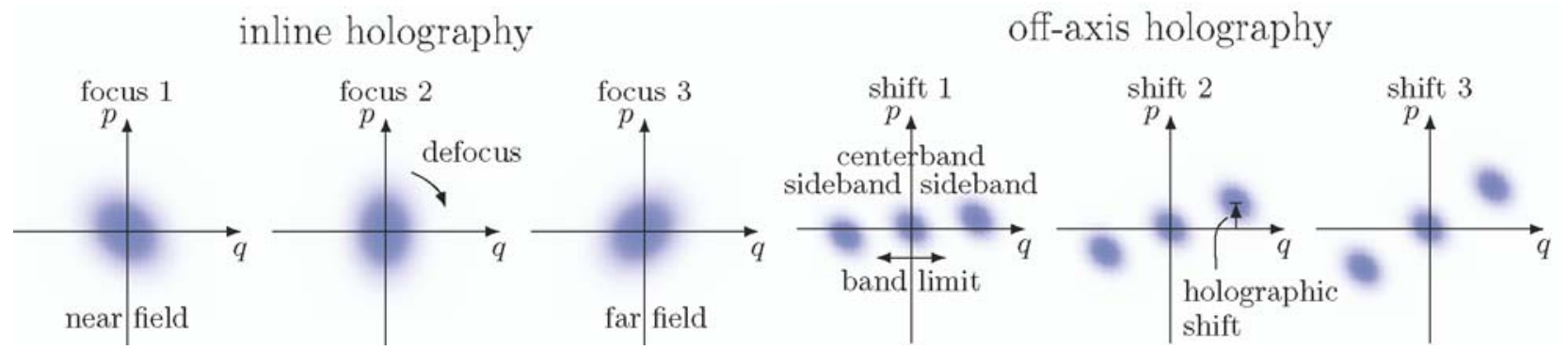

Figure 2. (Reciprocal) phase space diagrams of inline and off-axis holography. Some important prerequisites of the corresponding setup, such as the band-limit of the quantum state in off-axis EH or the defocus interval from the near to the far field in inline holography are indicated. The holographic shift is the distance between object and reference region adjusted by the biprism voltage. 